\title{
Ankle fractures in Finland: 118,929 operatively treated between 1987 and 2019
}

\author{
Ville HAPPONEN ${ }^{1}$, Heikki KRÖGER ${ }^{1,2}$, Markku KUISMIN ${ }^{2}$, and Reijo SUND ${ }^{2}$
}

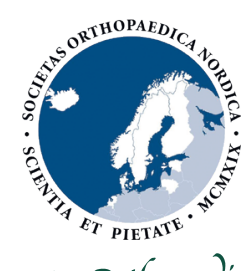

Acta Orthopaedica

\author{
${ }^{1}$ Department of Orthopedics, Traumatology and Hand Surgery, Kuopio University Hospital, Kuopio; ${ }^{2}$ Kuopio Musculoskeletal \\ Research Unit, University of Eastern Finland, Kuopio; University Hospital, Kuopio, Finland \\ Correspondence: ville.happonen@kuh.fi \\ Submitted 2021-09-14. Accepted 2022-01-19.
}

Background and purpose - Ankle fractures are one of the most common fractures requiring operative treatment. They occur most commonly in postmenopausal women and younger men and recent studies suggest that the incidence of ankle fractures is increasing. In this registry study, we analyze inpatient data of operatively treated ankle fractures during a period of 33 years from our comprehensive nationwide register data.

Patients and methods - The data on operatively treated ankle fracture patients between January 1, 1987 and December 31, 2019 was collected from the Finnish National Hospital Discharge Register and analyzed.

Results $-118,929$ ankle fractures were treated operatively. These included lateral malleolar fractures (33\%), bior trimalleolar fractures $(51 \%)$, medial malleolar fractures (7\%), and other fractures (9\%). Mean age was 53 (SD 17) years for women and 43 (16) for men. The average annual incidence rate was 69 per 100,000 person-years. Over the past 3 decades incidence has leveled off for women and has started to decline for men. However, the incidence is increasing in the elderly women group (over 70 years of age). In the over 50 age group, comorbidities have increased over the years, being more common in men than in women. The incidence of ankle fractures was higher during the winter months (November-March).

Interpretation - The number of operatively treated ankle fractures has leveled off during the last 33 years. However, nowadays we operate on more difficult fractures in elderly patients with comorbidities.
Ankle fractures are one of the most common fractures requiring operative treatment. The incidence of ankle fractures is estimated at 179-187 per 100,000 person-years $(\mathbf{1 , 2})$. In the UK data, ankle fractures are the third most common hospitalrequiring fracture after hip and wrist fractures (3).

Ankle fractures are most common in postmenopausal women and younger men, and the annual incidence increases in wintertime (2). In a large Swedish registry study (1987-2004), the incidence of ankle fractures treated in hospital was 71 per 100,000 person-years, and $83 \%$ of fractures required operative treatment (4). In previous studies, the incidence of ankle fractures increased $(5,6)$, which was considered to be due to an aging population and increased recreational activities (7).

We assume that, as the population ages, the proportion of older people with more comorbidities and more difficult fractures will increase. In addition, according to current knowledge, some of the ankle fractures that have been treated operatively before can now be treated nonoperatively (8-10), thus the number of surgeries could be expected to be declining.

We analyzed the incidence, annual trend, age and sex distribution, associated comorbidity, and seasonal variation of operatively treated ankle fractures in Finland between 1987 and 2019.

\section{Patients and methods}

This register study was performed on the whole population of Finland $(5,525,292$ inhabitants on the last day of 2019) from 1987 to 2019.

We identified all ankle fracture patients who were treated operatively in Finnish hospitals between January 1, 1987 and December 31, 2019 from the Finnish National Hospital Discharge Register (known as the Care Register for Health Care 
since 1994), which is maintained by the Finnish Institute for Health and Welfare. It provides data on age, sex, domicile of the subject, duration of hospital stays, primary and secondary diagnosis, and surgical procedures performed during the hospital stay. The register contains data on procedures performed in public and private hospitals, and the quality of the data is good (11). We gathered age- and sex-group specific population sizes from Statistics of Finland's (StatFin) online services, which is maintained by Statistics Finland. In particular, we used the annual mean population information according to age and sex while calculating annual incidences.

The identification of operatively treated ankle fractures was based on the combination of diagnosis and operation codes. We used fracture codes that referred to ankle fractures (fractures of the distal tibia, distal fibula, or both) and operation codes, which included ankle fracture open or closed reduction, and internal fixation or external fixation (for example, Hoffman external fixation).

For the years 1987-1996, we used the ICD 9 codes $8240 \mathrm{~A}-8249 \mathrm{~A}$, and the hospital union procedure nomenclature codes 9128 (open reduction and internal fixation), 9126 (closed reduction and internal fixation), and 9130 (external fixation). For the years 1996-2019, we used ICD10 codes S82.3-S82.9 and NOMESCO procedure codes NHJ10 (internal fixation of fracture of ankle), NHJ12 (internal fixation of fracture of ankle using bioimplant), and NGJ70 (external fixation of fracture of lower leg). We divided the ankle fractures (operated on with specific procedure codes) into 4 classes: Medial malleolar fractures consist of ICD-9 codes $8240 \mathrm{~A}-8241 \mathrm{~A}$ and ICD-10 code S82.5. Fractures of the lateral malleoli consist of ICD-9 codes 8242A-8243A and ICD 10 code $\mathrm{S} 82.6$. Bi- and trimalleolar fractures consist of ICD-9 codes $8244 \mathrm{~A}-8247 \mathrm{~A}$ and ICD-10 codes S82.7-S82.8 and other ankle fractures ICD $98248 X-S 249 X$ and ICD10 codes S82.3, S82.4, and S82.9.

We stratified register-based numbers of operations by year, age group (0-19, 20-49, 50-69, and over 70 years), sex, and season (summer/winter). We calculated the incidence of operatively treated ankle fractures per 100,000 person-years in total, for genders and by age groups. To illustrate the continuous smoothed trend from stratified values, we used the locally estimated scatterplot smoothing (LOESS) technique.

We examined patients' comorbidities according to the CCI, which was designed to predict 1-year mortality on the basis of a weighted composite score (12). It has been adapted and validated to work also with Finnish register data (13).

\section{Statistics}

The Poisson regression model was used to obtain incidence rate ratios (IRR) of the considered risk factors (age group, sex, season (summer/winter), calendar year). The numbers of fractures as well as the follow-up times in person years (approximated by corresponding population figures in Finland) were tabulated according to the used stratification. A logarithm of the follow-up time was included as an offset to the model, as we modeled the incidence and not counts. Because the length of the winter and summer months were not equal, the follow-up time in each year for every age, season, and sex group was weighted according to the number of winter and summer months before the analysis. Year was used only for adjustments, and was modeled by using natural splines to account for a potentially non-linear effect. To mitigate the effect of over-dispersion, we used White's sandwich estimator to obtain the robust standard errors and calculated the p-values together with the $95 \%$ confidence intervals accordingly.

We used the residual deviance (chi-square test) to evaluate our model and ended up using robust standard errors to compensate for overdispersion and natural splines for modelling calendar year. In any case, we used Poisson regression to calculate IRRs for a limited number of covariates, i.e., it was more a technical tool for adjusted IRR calculations than a real statistical model. The seasonal average number of operatively treated ankle fractures is presented with the $95 \%$ confidence interval (CI).

\section{Ethics, funding, and potential conflicts of interest}

The ethics review committee of the Hospital District of Northern Savo approved the study plan (26.6.2018: 852/2018 [PSSHP]). Data and permission to use the data were obtained from the Finnish Institute for Health and Welfare (Dnro THL/1620/5.05.00/2018). Due to privacy issues the use of data is restricted and cannot be shared. This work was supported by State Funding for Hospitals. The authors declared no conflicts of interest.

\section{Results}

Between 1987 and 2019, 118,929 ankle fractures were operatively treated in Finland. Of these, lateral malleolar fractures numbered $33 \%$, bi- or trimalleolar fractures $51 \%$, there were $7 \%$ medial malleolar fractures, and other fractures numbered $10,159(9 \%)$. For both sexes, the annual incidence increased towards the turn of the 21 st century, after which the incidence in women leveled off and in men began to decline (Figure 1).

The peak incidence in women was 122 per 100,000 person-years in the 50-69 years age group and in men 92 per 100,000 person-years in the $20-49$ years age group. The incidence of bi- or trimalleolar ankle fractures in women over 70 years of age has increased from 27 to 61 per 100,000 in three decades, with a more moderate increase in men, from 17 to 26 per 100,000. In contrast, the incidence of bi- or trimalleolar ankle fractures in 50-69-year-olds has remained stable in both women and men. The incidence decreased most in men aged 20-49 years (Table 1, Figure 2).

The seasonal variation is shown in Figure 3. The largest daily peaks are in the winter months (November-March). An average of 241 (95\% Cl 212-273) ankle fractures were 


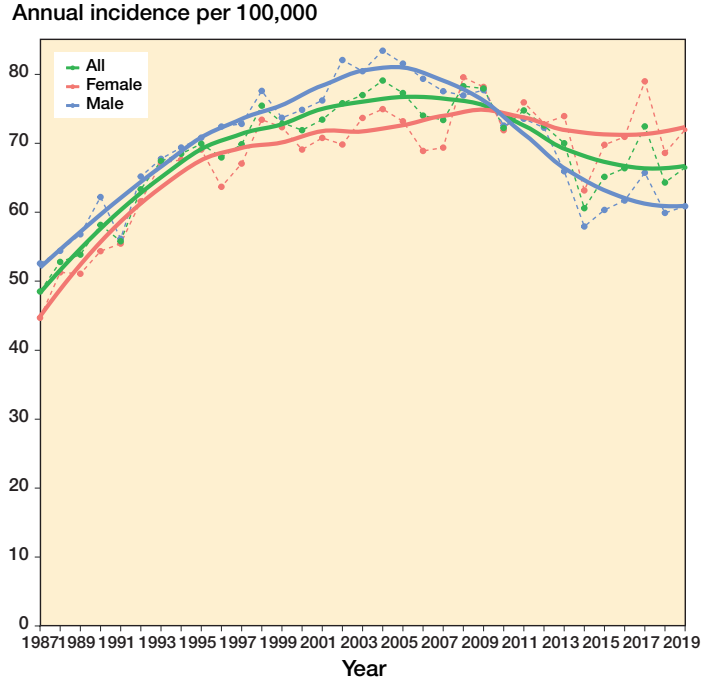

Figure 1. Ankle fractures incidence per 100,000 between 1987 and 2019. Solid lines illustrate the LOESS curves through data points with $50 \%$ smoothing span.

Incidence rate per 100,000 person-years
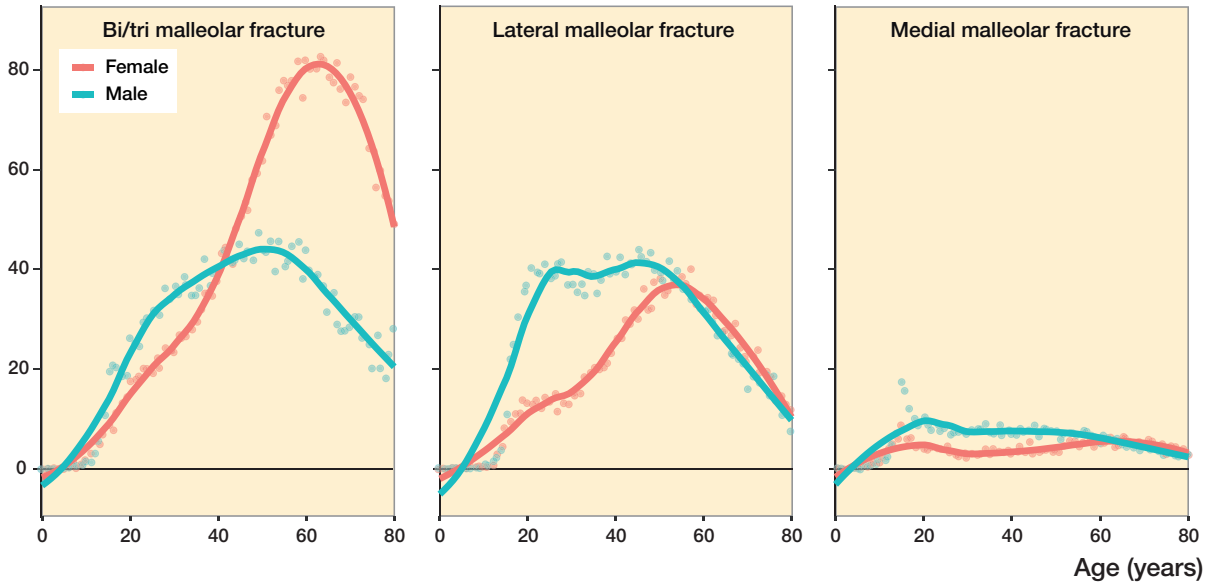

Figure 2. Incidence rates per 100,000 person-years of different ankle fractures during 1987-2019 for both genders plotted against age. Note that only persons of age 80 or under are shown in the plot. Solid lines illustrate the LOESS curves through data points with $50 \%$ smoothing span.

Table 1. Age and sex demographics of different fracture types. Values are count $(\%)$ unless otherwise specified

\begin{tabular}{lccccc}
\hline & $\begin{array}{c}\text { Bi-/tri } \\
\text { malleolar } \\
\text { fracture }\end{array}$ & $\begin{array}{c}\text { Lateral } \\
\text { malleolar } \\
\text { fracture }\end{array}$ & $\begin{array}{c}\text { Medial } \\
\text { malleolar } \\
\text { fracture }\end{array}$ & $\begin{array}{c}\text { Other } \\
\text { fracture }\end{array}$ & p-value \\
\hline Total & $60,174(51)$ & $40,130(34)$ & $8,466(7)$ & $10,159(9)$ & \\
Mean age (SD) & $51.0(17)$ & $45.2(16)$ & $41.7(19)$ & $44.9(19)$ & $<0.001$ \\
Age group & & & & & \\
$\quad$ 0-19 & $2,119(4)$ & $2,097(5)$ & $1,417(17)$ & $1,284(13)$ & $<0.001$ \\
20-49 & $24,879(41)$ & $21,643(54)$ & $3,966(47)$ & $4,469(44)$ & \\
$50-69$ & $24,256(40)$ & $13,627(34)$ & $2,348(28)$ & $3,451(34)$ & \\
$>70$ & $8,920(15)$ & $2,763(7)$ & $735(9)$ & $955(9)$ & \\
Sex & & & & & \\
$\quad$ Male & $24,336(40)$ & $23,248(58)$ & $5,149(61)$ & $5,999(59)$ & $<0.001$ \\
Female & $35,838(60)$ & $16,882(42)$ & $3,317(39)$ & $4,160(41)$ & \\
\hline
\end{tabular}

operatively treated in the summer months and $383(95 \% \mathrm{Cl}$ 346-423) in the winter months.

Chronic diseases such as diabetes, chronic lung disease, and malignancies emerge as underlying diseases. Comorbidities increase with age. In the elderly group, comorbidities are more common in men than in women. In the 50-69 and over 70 age groups, comorbidities have increased over the years (Figure 4, Table 2, see Supplementary data).

Based on the estimated coefficients of the Poisson regression model, the incidence rate ratio (IRR) for men is $2 \%$ higher compared with women, and people aged 50-69 have a twofold risk compared with those aged 20-49 years (Table 3 ). The IRR is $62 \%$ higher in the winter months. We decided to leave children out of this Poisson regression analysis because their fractures and treatment are different. However, differences in the incidences in all age groups can be viewed in Figure 6 (see. Supplementary data).

\section{Discussion}

Our study of all operatively treated ankle fractures in Finland over a period of 33 years shows that the incidence of operatively treated ankle fractures is leveling off in women, and in men has started to decline.

In women, more severe, bi- and trimalleolar fractures are more common, and the mean age is higher. In both sexes, the incidence is decreasing in the age group 20-49 years, steady in $50-60$-year-olds, and in $>70$ years is for men steady and for women increasing (Figure 5). This would suggest that ankle fractures are increasingly associated with fragility and osteoporosis. Similar views were presented by Rammelt in 2017 and Biver et al. in $2015 \mathbf{( 1 4 , 1 5 )}$. It must also be kept in mind that diagnostics and treatment of osteoporosis have evolved significantly during follow-up, and this may also affect the results.

Table 3. Estimated Poisson model coefficients, IRRs, robust $95 \%$ confidence interval $(\mathrm{rCl})$, and $\mathrm{p}$-value

\begin{tabular}{lcc}
\hline Factor & IRR $(\mathrm{rCl})$ & $\mathrm{p}$-value \\
\hline Male sex (ref. female) & $1.02(1.00-1.04)$ & 0.07 \\
Age group (ref. 20-49) & & \\
$\quad 50-69$ & $2.02(1.98-2.06)$ & $<0.001$ \\
$>70$ & $0.76(0.73-0.80)$ & $<0.001$ \\
Winter season (ref. summer) & $1.62(1.59-1.65)$ & $<0.001$ \\
\hline
\end{tabular}

Adjusted for year modeled with natural splines. 
Total number of fractures

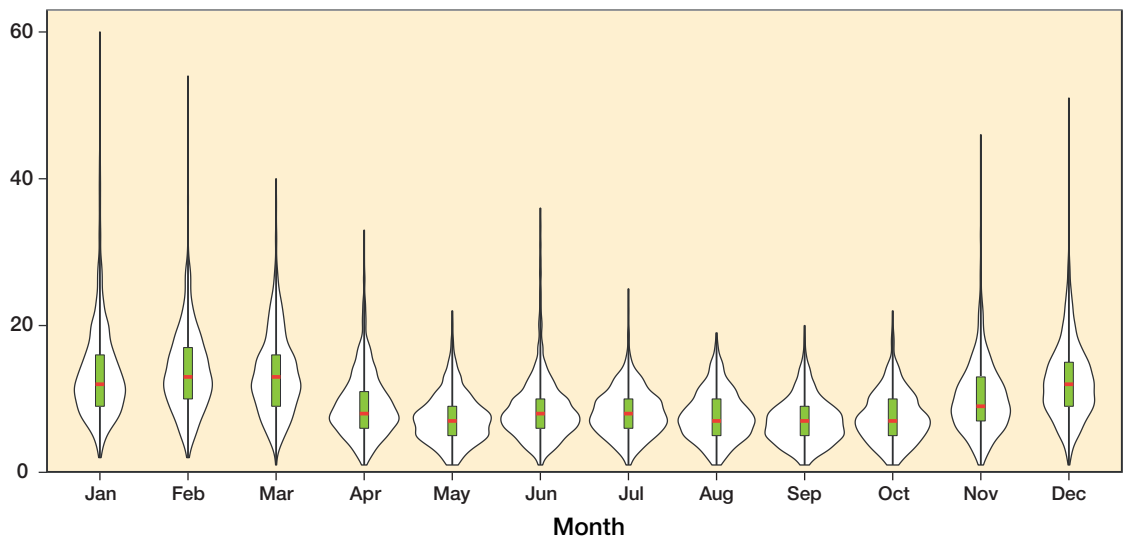

Figure 3. Average number of monthly fractures from 1987-2019 for both age and sex groups. The violin plots (outlines around the boxplots, which show the median and IQR) illustrate the estimated kernel probability density estimates of the monthly fractures; the wider the violin plot is the more observations have accumulated around that particular number of fractures.

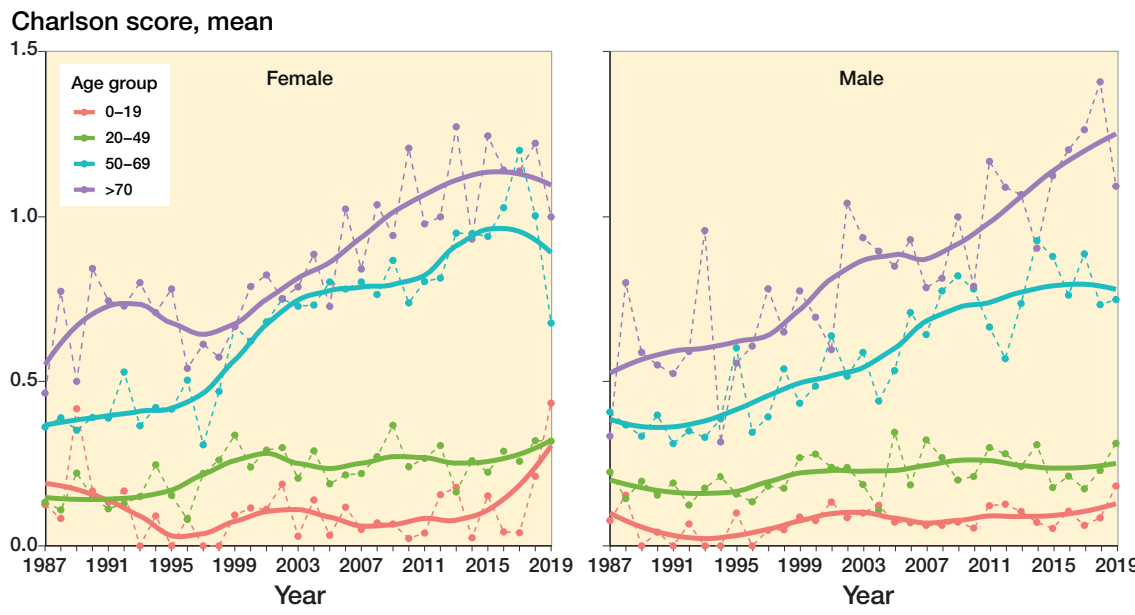

Figure 4. Average Charlson score from year 1987 to 2019 in each age group for females and males. Solid lines illustrate the LOESS curves through data points with $30 \%$ smoothing span.

\section{Incidence rate per 100,000 person-years}
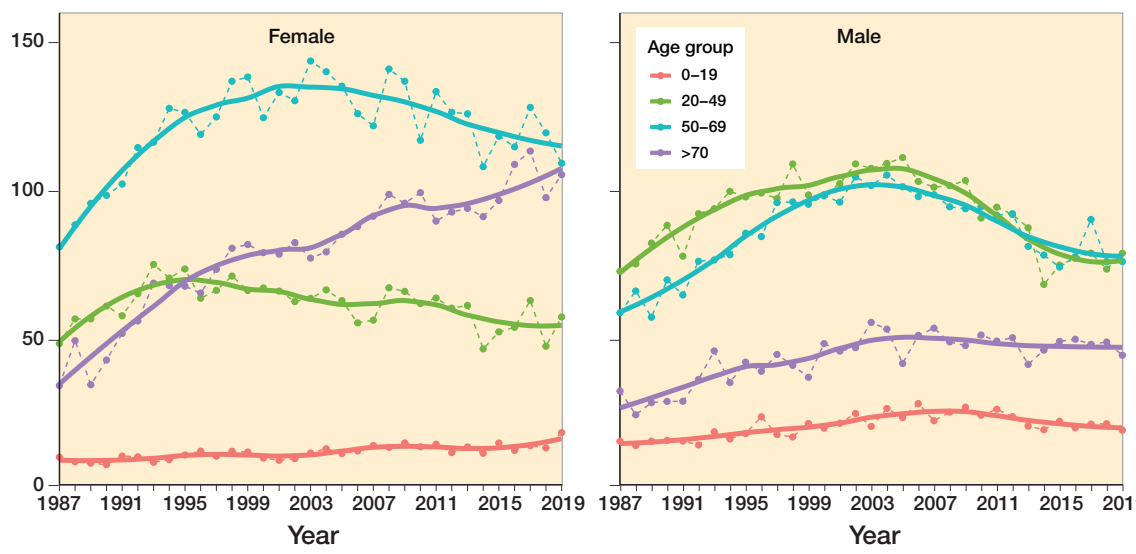

Figure 5. Ankle fracture incidence per 100,000 between 1987 and 2019 in each age group for females and males. Solid lines illustrate the LOESS curves through data points with $50 \%$ smoothing span.
The mean age in men is lower than in women, agreeing with previous studies (4). A new and interesting finding of our study is the clear decline in the incidence in men in the 20-49 age group, which could be explained by the more nonoperative treatment practice $(8,16,17)$. Furthermore, no polyclinic or outpatient clinic ankle fracture operations were performed in Finland during the follow-up period and most of the private sector ankle fracture operations are included in the register.

It is known that comorbidity increases with age and during the follow-up period Finland's population has increased by $11 \%$ and the proportion of the elderly (> 65 years) is also larger than before. In that sense, it is expected that comorbidities have increased over the years, especially in the elderly, but the change can also be seen in the age group 50-69 years. The explanation for the change may be that we now operate on sicker patients than before because it is safer with more advanced surgical techniques. Another explanation is that we are becoming better at diagnosing and registering comorbidities; the coverage of registers has also been improved. Although surgical techniques have evolved over the years, it remains to be seen whether the number of complications will increase.

In 2012, Thur et al. (4) published an epidemiological study of ankle fractures in Sweden from 1987-2004. In their study, the incidence of ankle fractures beginning to level off in the 21 st century and the annual increase was low $(0.2 \%)$. In Swedish data, $83 \%$ of ankle fractures treated in hospital were operated on. The mean age of women was 58 years, with the most typical being a bi-/trimalleolar fracture $(57 \%)$. The mean age of men was 45 years, and the most typical fracture was of the lateral malleoli (49\%). The demographic and weather conditions in our neighboring country are similar, so the study can be considered comparable. In our study the average age of Finnish women was 53 years, which is lower than in their study, and the most common type of fracture was bi- or trimalleolar $(59 \%)$, as in Swedes. In men, the mean age was 43 years, which is lower than in Sweden, and the most common type of fracture is a bi- or trimalleolar fracture (41\%), although lateral 
malleoli fractures account for $40 \%$ of fractures. Our study included only operatively treated ankle fractures, and this can explain differences in percentages of lateral fractures in men and younger ages in both sexes.

In 2016, Kannus et al. (18) reported that ankle fractures related to low-energy falls in the elderly ( $>60$ years) increased sharply between 1970 and 1997, after which the incidence of hospitalized ankle fractures decreased steadily until 2014. Our study includes more recent data up to 2019 and includes all age groups as well as more detailed information on fracture patients.

In 2018, Juto et al. (2) in a study from northern Sweden on the epidemiology of adult ankle fractures found that they were often caused by low-energy injury, and were more common in women. Annually, incidence increased when the mean temperature was close to 0 degrees Celsius and with roads and sidewalks at their most slippery. In our study, similar changes can be seen with the incidence increasing during NovemberMarch. During these months, in Finland average temperatures are 0 degrees Celsius or below and the majority of slip accidents occur. In addition, their study considers whether an ankle fracture can be classified as osteoporotic or not. A higher frequency in women, caused by low energy of injury, and an increase in incidence with age would suggest the signs of osteoporotic fracture (2). In previous studies, ankle fractures were not associated with osteoporosis $(19,20)$. However, in 2015, Biver et al. (15) showed an association between lower bone mineral density and ankle fracture. Our study supports the idea that an ankle fracture is becoming a fragility fracture in older people with poorer bone density.

Partio et al. (21) in a study (1996-2016) found that operatively treated ankle fractures are most common in young men and elderly women. The total incidence of operatively treated ankle fractures has decreased. This is, of course, one conclusion but on closer inspection total numbers appear to be leveling off in women and declining more clearly in men. In their study, one explanation for the reduction in operative treatment of ankle fractures was considered to be a more nonoperative treatment practice; stable ankle fractures can be treated nonoperatively. According to current knowledge, more than half of Weber B fractures are stable and can be treated nonoperatively with a few weeks of plaster or orthosis $(\mathbf{8 , 1 7 , 2 2 )}$. According to our study, the total number of operatively treated ankle fractures would appear to have started to decline in the early 2000s. One explanation for this change could be that the primary treatment of ankle fractures has shifted more to primary healthcare in the 21 st century and become more nonoperative in that sense.

To our knowledge, our study is one of the largest on operatively treated ankle fractures. The strength of our study is the long calculation of a time series and the data collected from a high-quality comprehensive national register, which covers the whole of Finland. Furthermore, the coverage and reliability of the Finnish National Hospital Discharge Register data is good $(11,23)$. Another strength of our study is that we also studied patients' comorbidities and looked at seasonal variation. Weaknesses in the study include changes in classifications and registers over time. Despite the changes, the numbers behave consistently and as expected, suggesting that the results are reliable. A weakness in our study is that in ICD-10 classification there is no specific code for bi- and trimalleolar fractures. S82.7 and S82.8 refer mostly to those fractures. ICD-10 codes S82.3 and S82.9 were also included in this study because they were recorded extensively along with the operation code NHJ10 (internal fixation of the ankle fracture), which is primarily the operation code for the ankle fractures NHJ12 (internal fixation of fracture of ankle using bioimplant) and NGJ70 (external fixation of fracture of lower leg). We considered a combination of diagnosis and operation codes to mostly represent ankle fractures, although for example combination S82.3 + NGJ70 may contain some Pilon fractures. In any case, diagnoses in those classes without relevant operation code were excluded. Another weakness is that, in such long-term follow-up, indications for ankle fracture surgery vary based on both standard practice and alternatives.

In summary, although people are aging and have more active lifestyles, operatively treated ankle fracture incidence has remained steady in the 2000s. However, the incidence of ankle fracture in elderly patients is increasing and these patients have more comorbidities, which may lead to more severe fractures and increased risk of complications.

VH: collection; analysis and interpretation of data; design of the study; and drafting of the manuscript. HK: interpretation of data; design of the study; and revision of the manuscript. RS: collection and interpretation of data; design of the study; and revision of the manuscript. MK: statistical work.

Acta thanks Charlotte K Thur and Svend Erik Østgaard for help with peer review of this study.

1. Daly P J, Fitzgerald R H Jr, Melton L J, Ilstrup D M. Epidemiology of ankle fractures in Rochester, Minnesota. Acta Orthop Scand 1987; 58 : 539-544. doi: 10.3109/17453678709146395.

2. Juto H, Nilsson H, Morberg P. Epidemiology of adult ankle fractures: 1756 cases identified in Norrbotten County during 2009-2013 and classified according to AO/OTA. BMC Musculoskelet Disord 2018; 19:441-x. doi: 10.1186/s12891-018-2326-x.

3. Jennison T, Brinsden M. Fracture admission trends in England over a ten-year period. Ann R Coll Surg Engl 2019; 101: 208-14. doi: 10.1308/ rcsann.2019.0002.

4. Thur C K, Edgren G, Jansson K A, Wretenberg P. Epidemiology of adult ankle fractures in Sweden between 1987 and 2004: a populationbased study of 91,410 Swedish inpatients. Acta Orthop 2012; 83: 276-81. doi: 10.3109/17453674.2012.672091.

5. Kannus P, Palvanen M, Niemi S, Parkkari J, Järvinen M. Increasing number and incidence of low-trauma ankle fractures in elderly people: Finnish statistics during 1970-2000 and projections for the future. Bone 2002; 31: 430-3.

6. Bengnér U, Johnell O, Redlund-Johnell I. Epidemiology of ankle fracture 1950 and 1980. Increasing incidence in elderly women. Acta Orthop Scand 1986; 57: 35-7. doi: 10.3109/17453678608993211. 
7. Court-Brown C M, Caesar B. Epidemiology of adult fractures: a review. Injury 2006; 37: 691-7

8. Van Schie-Van der Weert E M, Van Lieshout E M, De Vries M R, Van der Elst M, Schepers T. Determinants of outcome in operatively and non-operatively treated Weber-B ankle fractures. Arch Orthop Trauma Surg 2012; 132: 257-63. doi: 10.1007/s00402-011-1397-z.

9. Sanders D W, Tieszer C, Corbett B, Canadian Orthopedic Trauma Society. Operative versus nonoperative treatment of unstable lateral malleolar fractures: a randomized multicenter trial. J Orthop Trauma 2012; 26: 129-34. doi: 10.1097/BOT.0b013e3182460837.

10. Mittal R, Harris I A, Adie S, Naylor J M, CROSSBAT Study Group. Surgery for Type B ankle fracture treatment: a Combined Randomised and Observational Study (CROSSBAT). BMJ Open 2017; 7: e013298013298. doi: 10.1136/bmjopen-2016-013298.

11. Sund R. Quality of the Finnish Hospital Discharge Register: a systematic review. Scand J Public Health 2012; 40: 505-15. doi: $10.1177 / 1403494812456637$.

12. Charlson M E, Pompei P, Ales K L, MacKenzie C R. A new method of classifying prognostic comorbidity in longitudinal studies: development and validation. J Chronic Dis 40:373-383, 1987.

13. Pylväläinen J, Talala K, Murtola T, Taari K, Raitanen J, Tammela T L, et al. Charlson Comorbidity Index based on hospital episode statistics performs adequately in predicting mortality, but its discriminative ability diminishes over time. Clin Epidemiol 2019; 11: 923-32. doi: 10.2147/CLEP.S218697.

14. Rammelt S. Management of ankle fractures in the elderly. EFORT Open Rev 2017; 1: 239-246. doi: 10.1302/2058-5241.1.000023.

15. Biver E, Durosier C, Chevalley T, Herrmann F R, Ferrari S, Rizzoli R. Prior ankle fractures in postmenopausal women are associated with low areal bone mineral density and bone microstructure alterations. 2015; Osteoporos Int 26: 2147-55. doi: 10.1007/s00198-015-3119-9.
16. Michelson J D. Fractures about the ankle. J Bone Joint Surg Am 1995; 77: 142-52. doi: 10.2106/00004623-199501000-00020.

17. Pakarinen H J, Flinkkil T E, Ohtonen P P, Ristiniemi J Y. Stability criteria for nonoperative ankle fracture management. Foot Ankle Int 2011; 32: 141-7. doi: 10.3113/FAI.2011.0141.

18. Kannus P, Niemi S, Parkkari J, Sievanen H. Declining incidence of fall-induced ankle fractures in elderly adults: Finnish statistics between 1970 and 2014. Arch Orthop Trauma Surg 2016; 136: 1243-6. doi: 10.1007/s00402-016-2524-7.

19. Seeley D G, Browner W S, Nevitt M C, Genant H K, Scott J C, Cummings S R. Which fractures are associated with low appendicular bone mass in elderly women? The Study of Osteoporotic Fractures Research Group. Ann Intern Med 1991; 115: 837-42. doi: 10.7326/0003-4819-11511-837.

20. Hasselman C T, Vogt M T, Stone K L, Cauley J A, Conti S F. Foot and ankle fractures in elderly white women: incidence and risk factors. J Bone Joint Surg Am 2003; 85: 820-4. doi: 10.2106/00004623200305000-00008.

21. Partio N, Huttunen T T, Maenpaa H M, Mattila V M. Reduced incidence and economic cost of hardware removal after ankle fracture surgery: a 20-year nationwide registry study. Acta Orthop 2020; 1-5. doi: 10.1080/17453674.2020.1733749.

22. Kortekangas T, Haapasalo H, Flinkkilä T, Ohtonen P, Nortunen S, Laine $\mathbf{H} \mathbf{J}$, et al. Three week versus six week immobilisation for stable Weber B type ankle fractures: randomised, multicentre, non-inferiority clinical trial. BMJ 2019; 364: k5432. doi: 10.1136/bmj.k5432.

23. Mattila V M, Sillanpaa P, Iivonen T, Parkkari J, Kannus P, Pihlajamaki H. Coverage and accuracy of diagnosis of cruciate ligament injury in the Finnish National Hospital Discharge Register 2008; 39: 1373-6. doi: $10.1016 /$ j.injury.2008.05.007.

\section{Supplementary data}

Table 2. Sex demographics of Charlson comorbidities, which cover 118,929 incidences in total from year 1987 onward. Values are count (\%)

\begin{tabular}{lccc}
\hline Comorbidity & Male & Female & Total \\
\hline Chronic pulmonary disease & $876(15)$ & $1,407(16)$ & $2,283(16)$ \\
Diabetes with chronic complications & $471(8)$ & $561(6)$ & $1,032(7)$ \\
Any malignancy (including leukemia and lymphoma) & $766(14)$ & $1,545(18)$ & $2,311(16)$ \\
Diabetes without chronic complications & $982(17)$ & $1,205(14)$ & $2,187(15)$ \\
Congestive heart failure & $326(6)$ & $539(6)$ & $865(6)$ \\
Myocardial infarction & $367(6)$ & $301(3)$ & $668(5)$ \\
Cerebrovascular & $567(10)$ & $952(11)$ & $1,519(11)$ \\
Rheumatological disease & $163(3)$ & $783(9)$ & $946(7)$ \\
Hemiplegia or paraplegia & $49(1)$ & $76(1)$ & $125(1)$ \\
Peripheral vascular disease & $274(5)$ & $271(3)$ & $545(4)$ \\
Mild chronic liver disease & $331(6)$ & $271(3)$ & $602(4)$ \\
Moderate or severe renal disease & $144(3)$ & $178(2)$ & $322(2)$ \\
Dementia & $103(2)$ & $421(5)$ & $524(4)$ \\
Peptic ulcer disease & $182(3)$ & $163(2)$ & $345(2)$ \\
Metastatic solid tumor & $25(0.1)$ & $39(0.1)$ & $64(0.1)$ \\
Moderate or severe liver disease & $54(1)$ & $30(0.3)$ & $84(1)$ \\
\hline
\end{tabular}


Fractures per 100,000 - Females, 0-19 years

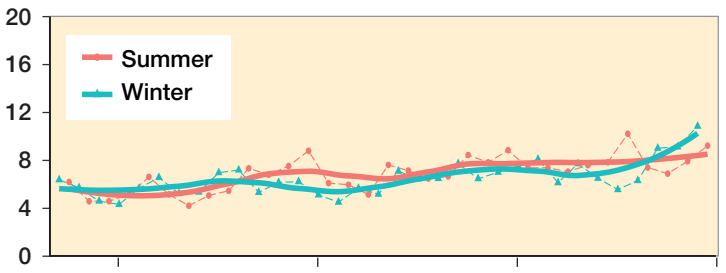

Fractures per 100,000 - Females, 20-49 years

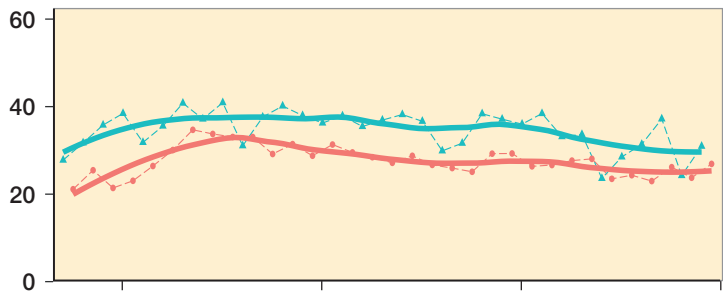

Fractures per 100,000 - Females, 50-69 years

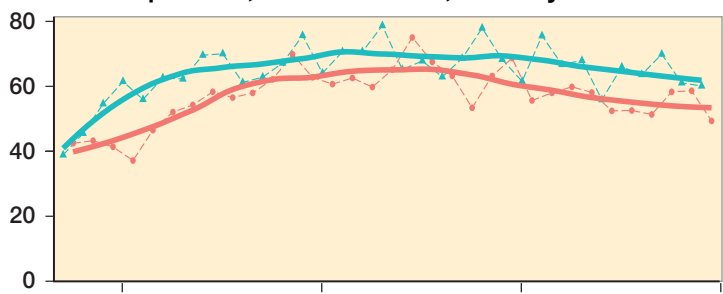

Fractures per 100,000 - Females, $>70$ years

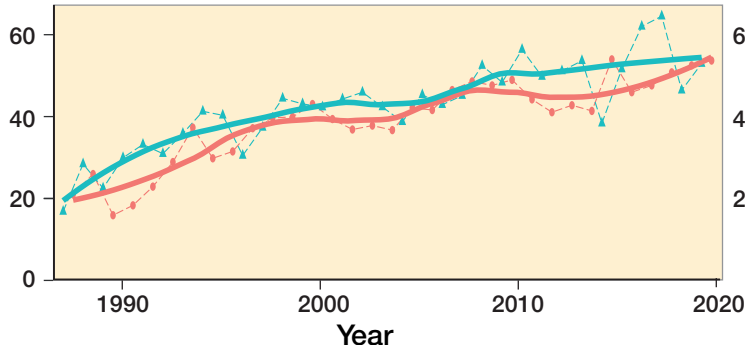

Fractures per 100,000 - Males, 0-19 years

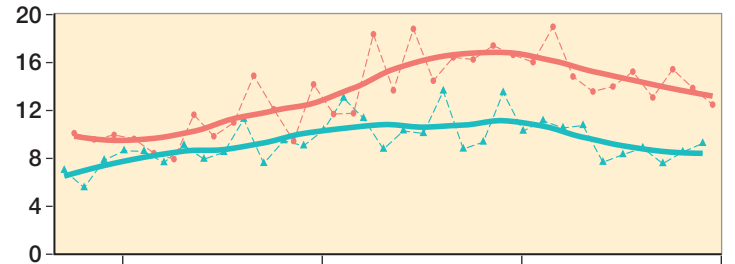

Fractures per 100,000 - Males, 20-49 years

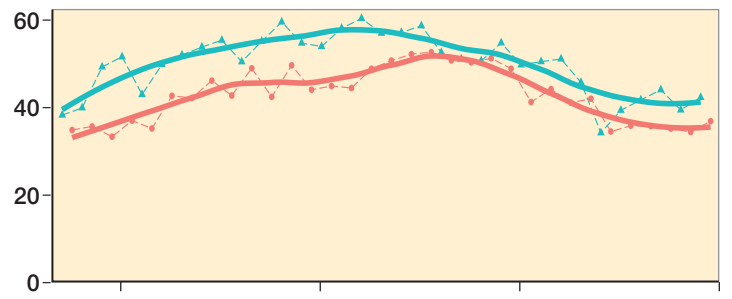

Fractures per 100,000 - Males, 50-69 years

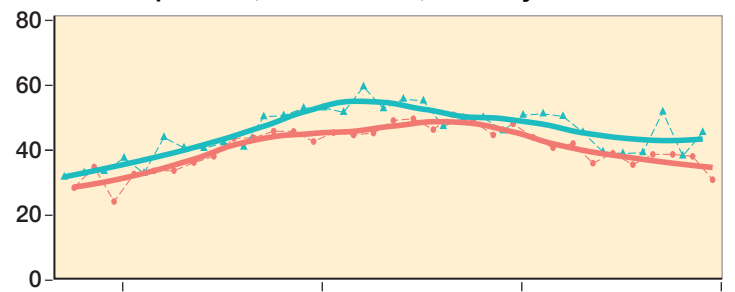

Fractures per 100,000 - Males, $>70$ years

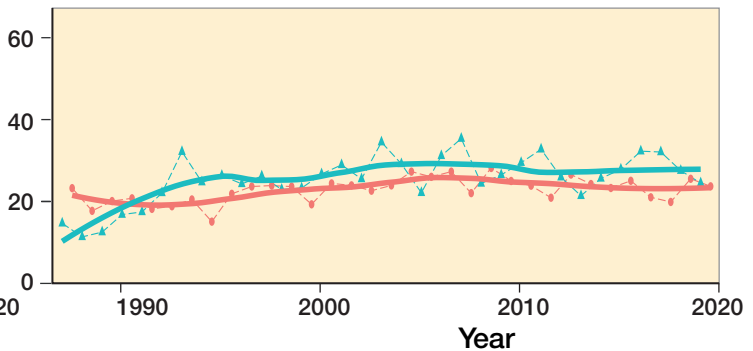

Figure 6. Incidence rate per 100,000 of seasonal fractures from 1987-2019 for both ages and sex groups. The solid lines illustrate the LOESS curves through data points with $50 \%$ smoothing span. 